\title{
Characterization of the adhesion force between avidin-functionalized AFM tips and biotinylated agarose beads
}

\author{
Markus Ludwig, Vincent T. Moy, Matthias Rief, Ernst-Ludwig Florin and Hermann E. \\ Gaub
}

Physikdepartment, Technische Universität Müchen, 85748 Garching, Germany

(Received July 4; accepted November 18, 1994)

\begin{abstract}
With AFM related techniques we investigated the specific interaction between avidin and biotin. The contact rupture force (adhesion) between avidin functionalized tips and biotin, which was covalently linked to agarose beads, was measured and characterized. A comprehensive study of the physical properties of the receptor-functionalized tips and the biotin-labeled agarose bead was carried out. The functional stability of the avidin coated tip persisted for several hundred force scan measurements. The strength of the interaction between the tip and the bead was found to depend on the force applied by the AFM tip on the polymer beads. By regulating the contact via indentation, the interaction can vary from one to as much as 100 biotin-avidin pairs. When surface interaction is minimized, quantized interaction between avidin and biotin was observed. This approach induces minimal stress on the interacting molecules.
\end{abstract}

\section{Introduction.}

During recent years the atomic force microscope (AFM) [1] has evolved into a versatile instrument for various experiments on the small length scale [2-6]. Particularly the option of this novel technique to operate under aqueous conditions and in real time has initiated a broad spectrum of new investigations $[7,8]$. As a result structural and micro mechanical properties of samples like molecular films [9-12], proteins [13, 14], supramolecular assemblies [15, 16] and even live cells [17-19] were elucidated. Recently specific biomolecular interactions were probed with the AFM [20-22] down to the resolution of individual molecular pairs [23]. Spring constants of individual polymers were measured (Moy et al. in prep). Here we have focused on the investigation of the specific interaction between biotin and streptavidin.

Avidin, a $67 \mathrm{kD}$ tetrameric protein, was initially identified as the component of egg white that induced nutritional deficiency in rat when given in excess amount [24]. Later, it was discovered that avidin binds to the $\mathrm{B}$ vitamin biotin, giving raise to the speculation that the biological role of avidin is that of an antibiotic scavenger. Although avidin has no direct medical benefits, it has found wide spread applications in diagnostic assays, biotechnology and medical science. The 
distinctive characteristic of avidin-biotin adhesion system that has generated considerable interest is its unique, practically irreversible, high affinity $\left(10^{15} \mathrm{M}^{-1}\right)$. Combined with the availability of structural and thermodynamic data, the avidin-biotin complex is an established model system for molecular recognition studies.

Recently, we introduced techniques developed for scanning probe microscopy to measure the interaction between avidin and biotin immobilized on opposing surfaces [23]. In these studies, avidin molecules were immobilized on AFM tips and biotin was covalently attached to a polymer matrix (see Fig. 1). As space was limited in our previous communications, only essential steps in the unbinding measurements were addressed. Here, we report a more thorough and comprehensive study of both the receptor functionalized tips and the target agarose substrate. These studies are deemed necessary for future endeavors. The specificity of ligand-receptor interaction combined with the spatial and force sensitivity of the AFM will undoubtedly fostered many applications.

\section{Materials and methods.}

AFM force measurements were obtained using a home-built stand-alone AFM with feedback electronics [25]. Visualized under an inverted microscope, the tip of AFM cantilever is positioned over the center of the biotin-labeled agarose beads, which rested on a sample chamber with a glass coverslip base. In the AFM force scan measurements, the interaction between AFM tips and samples is determined from changes in curvature of the cantilever. Our AFM uses the deflection of a laser spot off the back surface of the cantilever to monitor changes in the bent of the cantilever. During the initial phase of the scan, the interacting surfaces are brought in contact by the piezo element. In the case of elastic samples, further extension of the piezo causes the cantilever to press the tip into the sample and increases the area of interaction [26]. If the tip adheres to the sample, it remains in contact with the sample on retraction of the cantilever, resulting in the bending of cantilever towards the direction of the sample. The adhesive force of the system is operationally derived from the maximal bent of cantilever. The cantilever deflection was calibrated to piezo displacement in force scan measurements on glass surface. The spring constant of the cantilever $\left(k_{\mathrm{c}}=60 \mathrm{mN} / \mathrm{m}\right)$ was taken from the manufacture's specifications. All force values given in this paper are subject to the systematic error that is associated with this constant.

All measurements were carried out in phosphate buffer saline (PBS; $10 \mathrm{mM} \mathrm{PO}_{4}^{2-}$ and 150 $\mathrm{mM} \mathrm{NaCl}$ at $\mathrm{pH} 7.4)$ at room temperature $\left(20 \pm 2{ }^{\circ} \mathrm{C}\right)$. Biotin-labeled beads (agarose and acrylic) (Sigma, Deisenhofen, Germany) were washed three times in PBS and equilibrated in the same buffer for at least 10 minutes before use. NeutrAvidin (Pierce, Oud-Beijerland, Netherlands) is a deglycosylated, neutral form of avidin used in this study. $\mathrm{Si}_{3} \mathrm{~N}_{4}$ cantilevers were a generous gift from Digital Instruments (Santa Barbara).

\section{Results.}

Biotinamidocaproyl-labeled bovine serum albumin (biotin-BSA, Sigma) absorbed on AFM cantilevers was used as a linkage by which avidin is attached to an oriented on the tip, preventing avidin from directly interacting with the $\mathrm{Si}_{3} \mathrm{~N}_{4}$ surface and possibly, altering its binding characteristics. BSA also acts as a spacer to shield against van der Waals and attractive force between the interacting surface. In the initial phase of the investigations the BSA adsorption process produced highly variable results that could be correlated to the batch of cantilever used in the preparation. Apparently, the procedure for the fabrication of the cantilever has undergone many changes in attempts to produce tips that are sharper and more inert to non specific interaction with samples. 

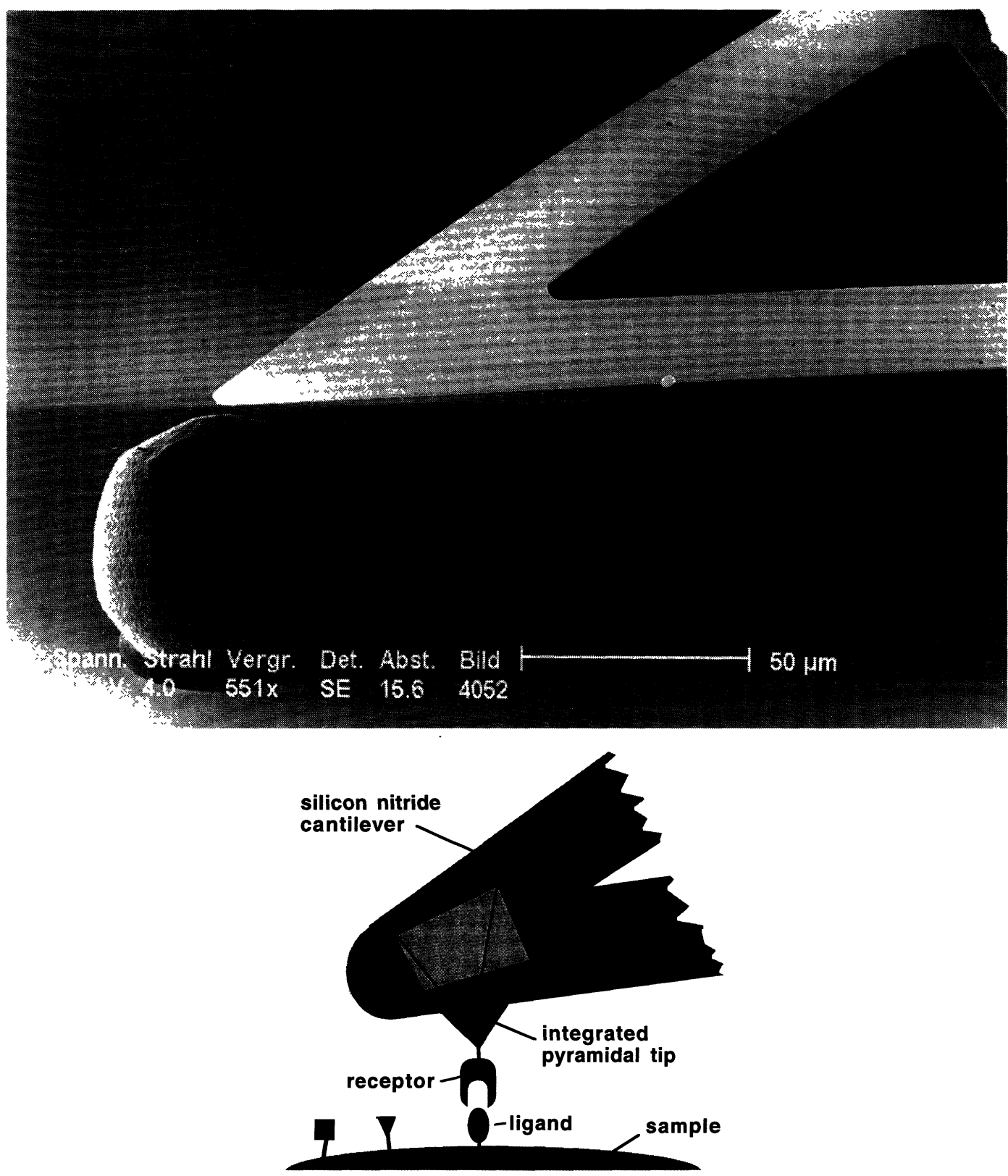

Fig. 1. - Electron micrograph a) and schematic drawing b) of an AFM cantilever and an agarose bead illustrating the experimental geometry.

This has resulted in recent preparations that demonstrate weaker BSA adsorption property. In our limited sampling, cantilevers from older wafers produced a much tighter adsorbed protein film.

The following protocol was adopted by our laboratory after experimenting with others (see Fig. 2). $\mathrm{Si}_{3} \mathrm{~N}_{4}$ cantilevers were irradiated with ultraviolet light (LOT Oriel 6035, Darmstadt, 

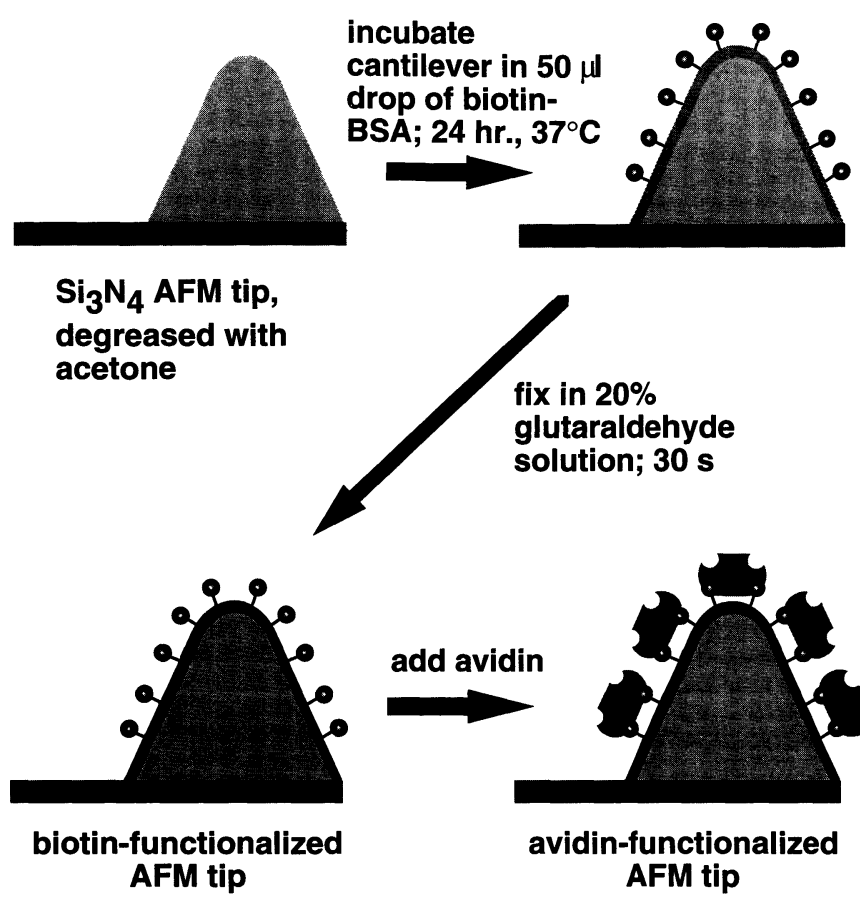

biotin avidin

Fig. 2. - Schematics of the preparation of the avidin-functionalized tips.

Germany) for 30 minutes and incubated in $50 \mu \mathrm{l}$ drop of biotin-BSA, previously resuspended in PBS and heat inactivated $\left(60^{\circ} \mathrm{C}\right)$, for 24 hours in a temperature controlled $\left(37^{\circ} \mathrm{C}\right)$ humidified chamber. After the adsorption of the biotin-BSA, the cantilevers were rinsed 3 times with PBS and dipped in a fresh $20 \%$ solution of glutaraldehyde (Sigma) for about 30 seconds to stabilize the protein film. The modified cantilevers were washed with PBS and stored at $4{ }^{\circ} \mathrm{C}$ until needed. Some tips remained active even one month after preparation. The attachment of the avidin was carried out just prior to an experiment. Tips were incubated in a $40 \mu \mathrm{l}$ drop of avidin $(1 \mathrm{mg} / \mathrm{ml})$ at room temperature for 5 minutes followed by extensive rinsing in PBS.

Measurements were carried out on biotin-labeled $4 \%$ agarose beads, a polysaccharide consisting of repeating units of galactose and 3,6-anhydro-L-galactose. The biotin molecules were coupled to the agarose via amide linkage formed between the carboxyl group of biotin and diaminospacer. Over a wide $\mathrm{pH}$ range, the matrix remained relatively neutral and displayed minimal adhesion with avidin-functionalized tips. The approach trace of force scan measurements on agarose beads revealed a soft, $100 \mathrm{~nm}$ thick, outer layer with $k_{\text {eff }} \approx 30 \mathrm{mN} / \mathrm{m}$ and stiffer inner core $\left(k_{\mathrm{eff}} \approx 5 \mathrm{~N} / \mathrm{m}\right)$. Measurements on the acrylic beads showed that the rigidity of substrate is beyond the sensitivity range of the instrument.

We have previously shown that avidin-functionalized AFM tips bind specifically to beaded agarose derivatized with biotin, but not to the substrate itself [20]. The adhesion was inhibited with either biotin which targets the tip or soluble avidin which recognizes biotin on the sample [23]. An important property of the tips in future applications is their stability. The functional stability of avidin-coated tips as defined by their ability to adhere to biotin-labeled beads, was 


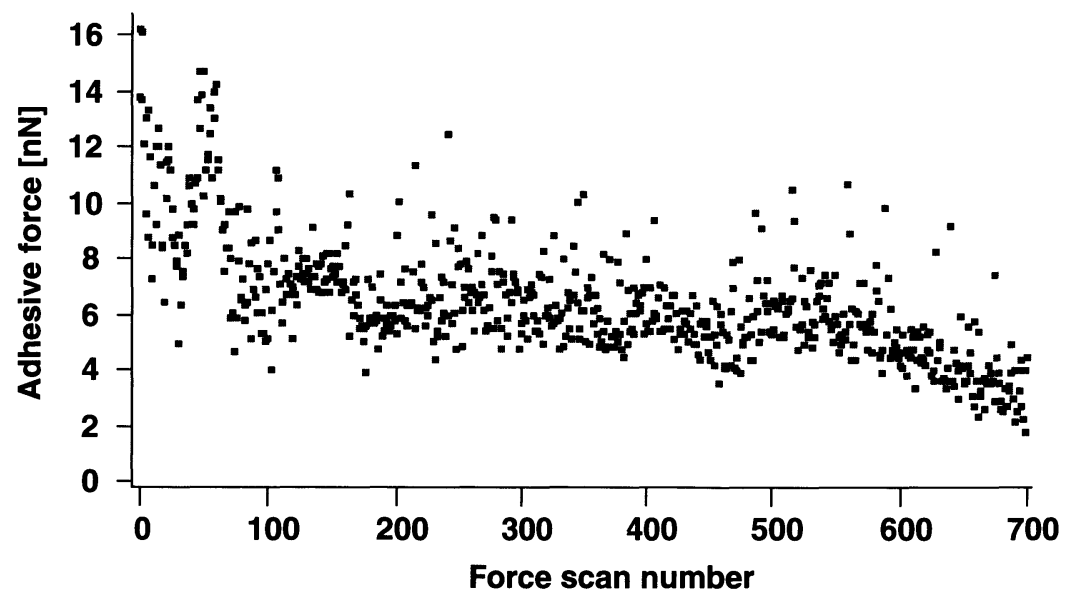

Fig. 3. - Stability of an avidin-functionalized AFM tip. The unbinding force of the avidin functionalized tip from the biotin agarose bead is plotted for repeated scans.

characterized by successive force scan measurements over the same spot on an agarose bead. As shown in figure 3, the avidin-functionalized tip retained functional activity after several hundred force scans. Typically, there is a rapid drop in the activity of the tip that can be attributed to loosely adsorbed biotin-BSA. The slower decay in functionality afterward may be due to i) the transfer of avidin to the biotin bead, ii) the structural breakdown of avidin, iii) the de-adsorption of biotinBSA from the tip, or it may reflect iv) the inhibition of avidin by biotin that dissociated from the beads. Impaired tips were treated with avidin in an attempt to rejuvenate function. Although activity increased slightly after avidin treatment, the activity declined very rapidly back to the values before treatment. This observation rules out the first possibility. However, further investigations will be needed to resolve this problem. If it is identified that biotin-BSA is coming off the tip then one possible solution is the direct covalent attachment of biotin to the silanol group of the tip surface. However, if the agarose filaments are being pulled out the bead, leading to poisoning of the tip, then one solution is to cross-link the beaded agarose.

The applied force exerted by the cantilever give raise to an indentation of the AFM tip into the elastic agarose bead. This indentation, in turn, results in an increase of the contact area between the interacting surfaces and subsequently, the strength of the interaction [6, 27]. Figure 4 summarizes adhesion measurements carried out under different indentation forces. In this series of experiment, the measurements were carried out first with increasing applied force, up to $24 \mathrm{nN}$, followed by a set of measurements done with decreasing applied force. The hysteresis in the two sets of measurements is attributed to a loss of functional activity of the tip. The adhesion of avidin-functionalized tip to biotin acrylic beads was significantly lower than to the biotin agarose beads. This is undoubtedly due to the difference in elasticity of the two substrates. By regulating the indentation force to less than $1 \mathrm{nN}$, the interaction between avidin tip and biotin beaded agarose can be lowered to a few molecules as evident in the histogram of rupture forces (Fig. 5). Two peaks, centered at 200 and $400 \mathrm{pN}$ corresponds to the interaction between one and two molecular pairs. Although the values are higher than the previously reported force quantum, it is within the calibration error of the cantilever. 


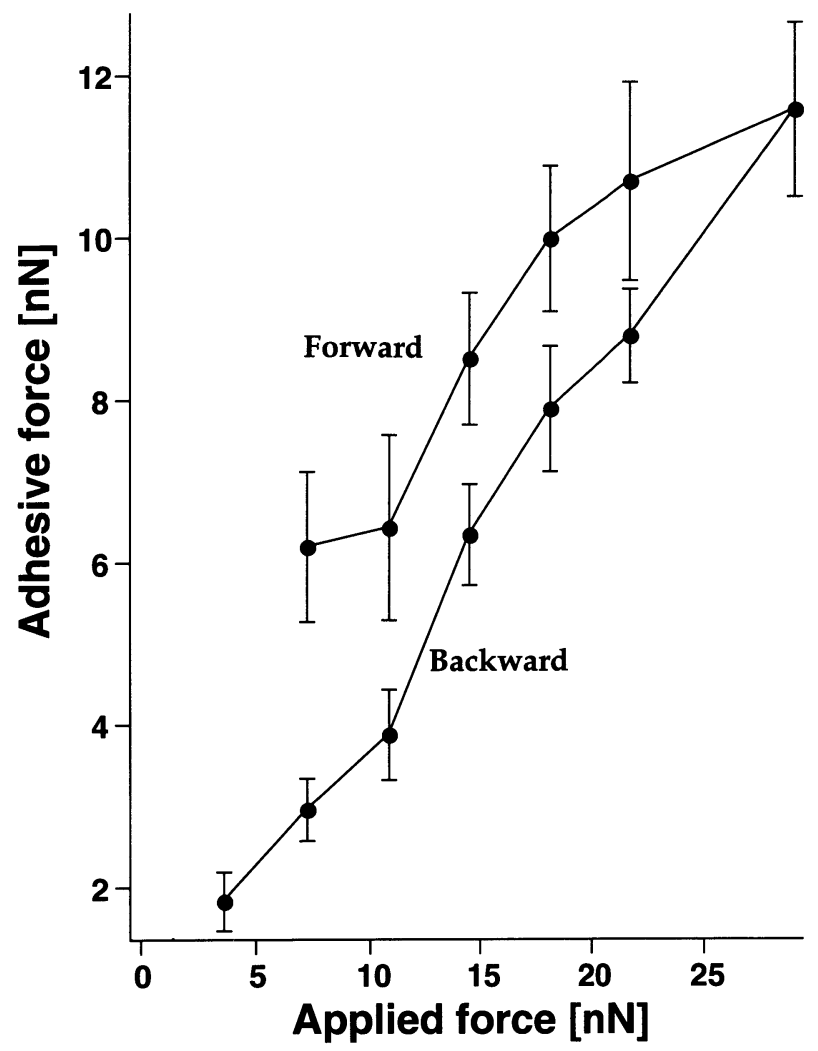

Fig. 4. - Adhesion of an avidin-functionalized tip to a biotin agarose bead as a function of the applied force.

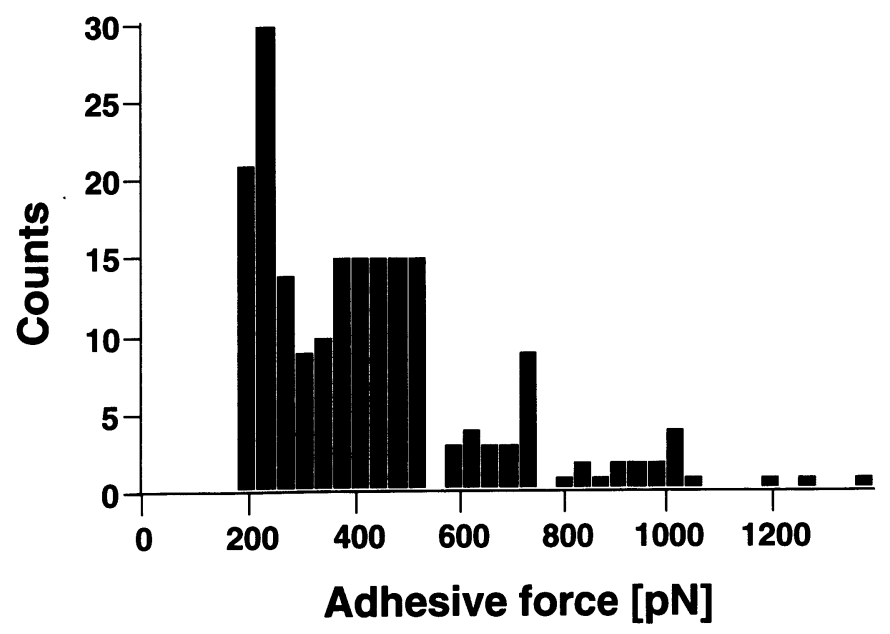

Fig. 5. - Histogram of the measured biotin-avidin rupture forces. 


\section{Discussion.}

The study reported here was carried out in light of the vast potential applications of surface modified AFM tips beyond force measurements between avidin and biotin. In previous reports, we demonstrated that by inhibiting the majority of the biotin molecules on the substrate with free avidin, it was possible to restrict the interaction between the surfaces to a few molecular pairs [20]. In this regime, the measured contact rupture forces were quantized, yielding the unbinding force of the adhesion system. In these early measurements, the AFM tips were pressed against the substrate with applied forces, typically 5 to $10 \mathrm{nN}$. Since the sample in our experiments is elastic, the force is spread over a larger area and the estimated pressure on the surface is 5 atmospheres. The decision to use this model system was partially motivated by the understanding that the avidin molecule can withstand high mechanical stress. SFA experiments have shown that avidin can tolerate pressures of up to 20 atmospheres [28]. Although the resulting pressure did not cause denaturation of the avidin molecules, these high pressure may potentially damage other proteins such as antibodies which are also potential candidates for this kind of investigations. Under these experimental conditions, the binding capacity of antibodies diminishes significantly after 10 measurements (data not shown, Moy et al., in prep.). To circumvent this complication, we have redesigned our measurements so that minimal contact was made during our measurements. The results demonstrates that discrete molecular recognition can be detected by this method.

\section{Acknowledgments.}

This work was supported by the Deutsche Forschungsgemeinschaft. Helpful discussions with E. Sackmann and G. Ahlefeld are gratefully acknowledged.

\section{References}

[1] Binnig G., Quate C.F. and Gerber C., “Atomic force microscope”, Phys. rev. lett. 56 (1986) 930.

[2] Drake B., Prater C.B., Weisenhorn A.L., Gould S.A.C., Albrecht T.R., Quate C.F., Channell D.S., Hansma H.G. and Hansma P.K., "Imaging crystals, polymers and biological processes in water with AFM", Science 243 (1989) 1586-1589.

[3] Hansma H.G., Vesenka J., Kelderman G., Morrett H., Sinsheimer R.L., Elings V., Bustamante C. and Hansma P.K., "Reproducible imaging and dissection of plasmid DNA under liquid wit the AFM", Science 256 (1992) 1180-1184.

[4] Ohnesorge F. and Binnig G., "True Atomic Resolution by Atomic Force Microscopy Through Repulsive and Attractive Forces", Science 260 (1993) 1451-1456.

[5] Manne S., Hansma P.K., Massie J., Elings V.B. and Gewirth A.A., "Atomic resolution electrochemistry with the atomic force microscope: copper deposition on gold surfaces", Science 251 (1991) 183.

[6] Radmacher M., Tillmann R.W. and Gaub H.E., "Imaging Viscoelasticity by Force Modulation with the Atomic Force Microscope", Biophys. J. 64 (1993) 735-742.

[7] Engel A., "Biological applications of scanning probe microscopes", Annu. Rev. Biophys. Biophys. Chem. 20 (1991) 79-108.

[8] Radmacher M., Tillmann R.W., Fritz M. and Gaub H.E., "From molecules to cells- imaging soft samples with the AFM", Science 257 (1992) 1900-1905.

[9] Egger M., Ohnesorge F., Weisenhorn A., Heyn S.P., Drake B., Prater C.B., Gould S.A.C., Hansma P. and Gaub H.E., "Wet lipid-protein membranes imaged at submolecular resolution by atomic force microscopy”, J. Struct. Biol. 103 (1990) 89-94. 
[10] Garnaes G., Schwartz D.K., Viswanathan R. and Zazadinski J.A.N., "Domain boundaries and buckling superstructures in LB films", Nature 257 (1992) 508-511.

[11] Tillmann R.W., Radmacher M., Gaub H.E., Kenny P. and Ribi H.O., "Monomeric and polymeric molecular films from diethylene glycol diamine pentacosadiynoic amide", J. Phys. Chem. 97 (1993) 2928-2932.

[12] Putman C.A.J., Hansma H.G., Gaub H.E., Greve J. and Hansma P.K., "Polymerized L-B films with a combined AFM-fluorescence microscope", Langmuir 8 (1992) 3014-3019.

[13] Karrasch S., Dolder M., Ramsden J., Schabert F. and Engel A., "Covalent binding of biological samples for scanning force microscopy”, Biophys. J. 65 (1993) 2437-2446.

[14] Weisenhorn A.L., Drake B., Prater C.B., Gould S.A.C., Hansma P.K., Ohnesorge F., Egger M., Heyn S.P. and Gaub H.E., "Immobilized proteins in buffer imaged at molecular resolution by atomic force microscopy”, Biophys. J. 58 (1990) 1251-1258.

[15] Schwinn T., Rabe J.P. and Gaub H.E., "Supramolecular Structures and Dynamics of Organic Adsorbate Layers at the Solid-Liquid Interface”, Surpramol. Sci. 2 (1994) in press.

[16] Manne S., Cleveland J.P., Gaub H.E., Stucky G.D. and Hansma P.K., "Direct Visualization of Surfactant Hemicelles by Force Microscopy of the Electrical Double Layer", Langmuir (1994) in press.

[17] Henderson E., Haydon P.G. and Sakaguchi D.S., "Actin filament dynamics in living glial cells imaged by atomic force microscopy", Science 257 (1992) 1944-1946.

[18] Häberle W., Hörber J.K.H., Ohnesorge F., Smith D.P.E. and Binnig G., "In situ investigations of living cells infected by viruses", Ultramicroscopy $42-44$ (1992) 1161-1167.

[19] Fritz M., Radmacher M. and Gaub H.E., "Granula motion and membrane spreading on human platelets imaged with the AFM", Biophys. J. 66 (1994) 1-7.

[20] Moy V.T., Florin E.-L. and Gaub H.E., "Adhesive forces between ligand and receptor measured by AFM", J. Colloids \& Surfaces.

[21] Lee G.U., Kidwell D.A. and Colton R.J., "Sensing discrete streptavidin-biotin interactions with AFM", Langmuir 10 (1994) 354-361.

[22] Pierce M., Stuart J., Pungor A., Dryden P. and Hlady V., "Specific and nonspecific adhesion force measurements using AFM with a linear position sensitive detector", Langmuir, submitted.

[23] Florin E.L., Moy V.T. and Gaub H.E., "Adhesion forces between individual ligand-receptor pairs", Science 264 (1994) 415-417.

[24] Green N.M., “Avidin”, Adv. Prot. Chem. 29 (1975) 85-133.

[25] Florin E.-L., Radmacher M., Fleck B. and Gaub H.E., "AFM with direct force modulation", Rev. Sci. Instr. 65 (1994) 639-643.

[26] Weisenhorn A.L., Khorsandi M., Kasas S., Gotozos V., Celio M.R. and Butt H.J., "Deformation and height anomaly of soft surfaces studied with the AFM", Nanotech. 4 (1993) 106-113.

[27] Hertz H., "Über den Kontakt elastischer Körper”, J. Reine Angew. Mathematik 92 (1881) 156.

[28] Helm C., Knoll W. and Israelachvili J., "Measurement of ligand-receptor interactions", PNAS 88 (1991) 8169-8173. 